\title{
Meteorological Parameters and the Onset of Chest Pain in Subjects with Acute ST-Elevation Myocardial Infarction: an Eight-Year, Single-Center Study in China
}

\author{
En-Zhi Jia ${ }^{a}$ Zhe Liu ${ }^{a}$ Ai-Jun Chen ${ }^{b}$ Yan Gu ${ }^{a}$ Zhao-Yang Lia Tie-Bing Zhu ${ }^{a}$ \\ Chun-Jian Lia Lian-Sheng Wang ${ }^{a}$ Wen-Zhu Ma ${ }^{a}$ Zhi-Jian Yanga \\ aDepartment of Cardiovascular Medicine, First Affiliated Hospital of Nanjing Medical University, \\ Nanjing, China; 'bSchool of Atmosphere Physics, Nanjing University of Information Science and \\ Technology, Nanjing, China
}

\section{Key Words}

Acute ST-elevation myocardial infarction - Atmospheric pressure variation • Temperature

\begin{abstract}
Objective: The purpose of this study was to investigate the influence of weather on the occurrence of acute ST-elevation myocardial infarction in Chinese subjects. Methods: Weather and climate data, as well as the occurrence of STEMI, were monitored at $2 \mathrm{am}, 8 \mathrm{am}, 2 \mathrm{pm}$, and 8 pm between 2003 and 2010. Generalized additive Poisson models were utilized to plot the numbers of patients with STEMI within 6 hour intervals against climatological variations, after accounting for the effects of the hour and season. Results: The inclusion of meteorological conditions, including observed atmospheric pressure ( $\mathrm{hPa}$, hectopascal) variations during the previous three hours and temperature $\left({ }^{\circ} \mathrm{C}\right.$, degrees Celsius), significantly affected the occurrence of STEMI, as measured every six hours. Compared with the 50th percentile of atmospheric pressure variations, the RRs $(95 \% \mathrm{Cl})$ for the first percentile, 10th percentile, 25th percentile, 75th percentile, 90th percentile, and 99th percentile of atmospheric pressure variation over lag 0 were 1.66 (1.36 2.03), 1.47 (1.30 1.67), 1.22 (1.12 1.33), 1.16 (1.07 1.25), 1.27 (1.13 1.43), and $1.16(0.92 \sim 1.46)$, respectively. Compared to the 50th percentile of temperature, the RRs $(95 \% \mathrm{Cl})$ for the first percentile, 10 th percentile, 25 th percentile, 75 th percentile, 90 th percentile, and 99th percentile of temperature over lag 0 were $0.58(0.40 \sim 0.83), 0.60(0.46 \sim 0.78), 0.69$ (0.57 0.83), 1.33 (1.14 1.56), 1.39 (1.13 1.71), and 1.17 (0.84 1.63), respectively. Conclusions: Based on the eight-year, single-center study, significant relationships were observed among the occurrence of STEMI and atmospheric pressure variations during the previous three hours and temperature after account for long-term time trends.
\end{abstract}

Z. Liu and E.-Z. Jia contributed equally to this article.

Dr. En-Zhi Jia

Department of Cardiovascular Medicine, First Affiliated Hospital of Nanjing Medical University, Guangzhou Road 300, Nanjing 210029, Jiangsu Province (China)

Tel. +86-139 51623205, Fax 0086-025-84352775, E-Mail enzhijiacn@aliyun.com 


\section{Introduction}

Acute ST-elevation myocardial infarction (STEMI), a clinical syndrome defined by characteristic symptoms of myocardial ischemia in association with persistent electrocardiographic (ECG) ST elevation and the subsequent release of biomarkers of myocardial necrosis [1], is a major cause of mortality and morbidity worldwide [2]. Recently, investigators have focused on the relationship between meteorological parameters and the occurrence of acute myocardial infarction (MI). A case-crossover study performed in the United States indicated that exposure to cold increased the risk of acute MI, and exposure to heat increased the risk of dying after an acute MI [3]. A Korean AMI Registry study found that the occurrence of AMI demonstrates seasonal and monthly variations and that meteorological parameters have a significant influence on the occurrence of AMI, particularly in females and in younger patients [4]. Additionally, a study performed in southern China indicated that cool temperatures and higher $\mathrm{NO}_{2}$ levels substantially increased the risk of AMI in these normally warm-climate cities: the sizes of the observed effects were larger than those noted in previous studies [5]. However, most of the studies in question have primarily examined the association between mortality or hospitalization due to acute myocardial infarction and meteorological parameters. Due to periodic variations in the time distribution patterns of the onset of chest pain in subjects with acute ST-elevation myocardial infarction [6], an exploration of the association between meteorological parameters and the time distribution patterns of the onset of chest pain in subjects with acute ST-elevation myocardial infarction is necessary.

The purpose of this study was to investigate the influence of weather on the occurrence of acute ST-elevation myocardial infarction in Chinese subjects and to use this information to develop a means of forecasting acute ST-elevation myocardial infarction occurrence in the future.

\section{Patients and Methods}

The study protocol was approved by the Ethics Committee of the First Affiliated Hospital of Nanjing Medical University (Nanjing, China). Written informed consent was provided by each patient.

\section{Study subjects}

This study was a prospective, observational single-center study that investigated the association between meteorological parameters and time distribution patterns in patients with STEMI, in China. Adult patients with acute STEMI who presented to the First Affiliated Hospital of Nanjing Medical University from 1 January 2003 to 31 December 2010 were consecutively enrolled in and included in the study. The diagnosis of acute STEMI was defined as $\geq 30 \mathrm{~min}$ of continuous chest pain, ST-segment elevation $>2.0$ $\mathrm{mm}$ on $\geq 2$ contiguous electrocardiographic leads, and a two-fold elevation or more in creatine kinase-MB (CKMB) levels [7]. Exclusion criteria included cardiac shock, severe liver or renal dysfunction, drug allergy, and severe hypovolemia. The rationale for the use of these criteria was that subjects suffering from the above complications may not have been able to accurately report the time of acute STEMI onset, which was determined by self-reported chest pain. Overall, 1467 subjects were included in this study.

\section{Climatological data}

Meteorological data are based on observations by the Meteorological Information Comprehensive Analysis and Process System (MICAPS) of China and were provided by the School of Atmosphere Physics, Nanjing University of Information Science and Technology.

Nanjing city is located at a latitude of $118^{\circ} 46^{\prime}$ East and longitude of $32^{\circ} 03^{\prime}$ North. The weather and climate data, including sea level pressure (HPA, hectopascal), atmospheric pressure variations during the previous three hours ( $\mathrm{hPa}$, hectopascal), six hour precipitation ( $\mathrm{mm}$, millimeter), dew-point temperature $\left({ }^{\circ} \mathrm{C}\right.$, degrees Celsius), visibility (km, kilometer), and temperature $\left({ }^{\circ} \mathrm{C}\right.$, degrees Celsius), were monitored at 2 am, $8 \mathrm{am}, 2 \mathrm{pm}$, and $8 \mathrm{pm}$. Meteorological data for November and December of 2007 and July of 2008 were not included. 
Table 1. Meteorological conditions and numbers of patients with STEMI for each 6 hour interval during the study period (from 1 January 2003 to 31 December 2010). SLP, Sea level pressure; APV, Atmospheric pressure variation during the previous three hours; Precipitation, Six hour precipitation; DPT, Dew-point temperature

\begin{tabular}{|c|c|c|c|c|c|c|c|c|c|c|c|}
\hline Variable & Mean & SD & Min & P1 & P10 & P25 & P50 & P75 & P90 & P99 & Max \\
\hline STEMI; 2 am & 0.09 & 0.303 & 0 & 0 & 0 & 0 & 0 & 0 & 0 & 1 & 2 \\
\hline $\operatorname{SLP}(\mathrm{hPa}) ; 2 \mathrm{am}$ & 181.46 & 142.69 & 0 & 6.00 & 46.00 & 85.00 & 168.00 & 247.00 & 308.00 & 983.00 & 999.00 \\
\hline APV $(\mathrm{hPa}) ; 2$ am & -4.93 & 8.08 & -30.00 & -23.00 & -14.00 & -10.00 & -5.00 & -1.00 & 5.00 & 19.00 & 65.00 \\
\hline Precipitation (mm); 2 am & 0.82 & 3.99 & 0.00 & 0.00 & 0.00 & 0.00 & 0.00 & 0.00 & 1.00 & 19.30 & 81 \\
\hline $\operatorname{DPT}\left({ }^{\circ} \mathrm{C}\right) ; 2 \mathrm{am}$ & 11.01 & 10.11 & -19.40 & -12.13 & -2.80 & 3.20 & 11.90 & 19.70 & 23.98 & 26.20 & 29.50 \\
\hline Visibility $(\mathrm{km}) ; 2$ am & 7.40 & 3.96 & 0 & 1.00 & 2.00 & 4.00 & 7.00 & 11.00 & 12.00 & 15.28 & 20 \\
\hline Temperature $\left({ }^{\circ} \mathrm{C}\right)$; $2 \mathrm{am}$ & 14.44 & 9.28 & -7.40 & -4.20 & 1.20 & 6.80 & 15.60 & 22.50 & 26.10 & 30.00 & 31.90 \\
\hline STEMI; 8 am & 0.14 & 0.399 & 0 & 0 & 0 & 0 & 0 & 0 & 1 & 2 & 3 \\
\hline $\operatorname{SLP}(\mathrm{hPa}) ; 8 \mathrm{am}$ & 182.94 & 123.46 & 1 & 13.00 & 52.00 & 94.00 & 176.00 & 251.00 & 310.00 & 479.45 & 999.00 \\
\hline $\mathrm{APV}(\mathrm{hPa},) ; 8 \mathrm{am}$ & 11.28 & 7.82 & -18.00 & -7.00 & 3.00 & 7.00 & 11.00 & 15.00 & 20.00 & 36.08 & 63.00 \\
\hline Precipitation (mm); 8 am & 0.87 & 4.67 & 0.00 & 0.00 & 0.00 & 0.00 & 0.00 & 0.00 & 0.80 & 21.00 & 94.00 \\
\hline DPT $\left({ }^{\circ} \mathrm{C}\right) ; 8 \mathrm{am}$ & 11.36 & 10.11 & -20.20 & -10.52 & -2.78 & 3.20 & 12.70 & 20.10 & 24.30 & 26.30 & 28.30 \\
\hline Visibility $(\mathrm{km}) ; 8 \mathrm{am}$ & 6.22 & 4.14 & 0 & 0.20 & 2.00 & 3.00 & 5.00 & 10.00 & 12.00 & 18.00 & 30.00 \\
\hline Temperature $\left({ }^{\circ} \mathrm{C}\right) ; 8$ am & 15.19 & 9.87 & -7.20 & -4.20 & 1.00 & 6.60 & 16.60 & 23.60 & 27.60 & 31.70 & 33.60 \\
\hline STEMI; 2 pm & 0.16 & 0.395 & 0 & 0 & 0 & 0 & 0 & 0 & 1 & 1 & 3 \\
\hline SLP (hPa); 2 pm & 171.40 & 140.69 & 0 & 6.00 & 40.40 & 81.00 & 157.00 & 232.00 & 291.00 & 986.00 & 999.00 \\
\hline $\mathrm{APV}(\mathrm{hPa}) ; 2 \mathrm{pm}$ & -18.71 & 9.42 & -62.00 & -41.00 & -30.00 & -25.00 & -18.00 & -13.00 & -7.00 & 5.00 & 23.00 \\
\hline Precipitation (mm); $2 \mathrm{pm}$ & 0.75 & 4.14 & 0 & 0.00 & 0.00 & 0.00 & 0.00 & 0.00 & 0.70 & 17.00 & 77.00 \\
\hline $\mathrm{DPT}\left({ }^{\circ} \mathrm{C}\right) ; 2 \mathrm{pm}$ & 9.58 & 10.97 & -23.40 & -16.58 & -5.30 & 1.60 & 10.40 & 19.20 & 23.50 & 26.00 & 27.50 \\
\hline Visibility $(\mathrm{km}) ; 2$ pm & 9.84 & 5.20 & 0 & 1.50 & 3.00 & 6.00 & 10.00 & 14.00 & 17.00 & 23.00 & 28.00 \\
\hline Temperature $\left({ }^{\circ} \mathrm{C}\right) ; 2 \mathrm{pm}$ & 20.37 & 9.68 & -2.70 & 0.62 & 6.20 & 12.40 & 22.20 & 28.20 & 32.40 & 36.48 & 40.00 \\
\hline STEMI; 8 pm & 0.11 & 0.337 & 0 & 0 & 0 & 0 & 0 & 0 & 0 & 1 & 3 \\
\hline SLP (hPa); $8 \mathrm{pm}$ & 175.27 & 133.01 & 0.00 & 7.00 & 44.00 & 85.00 & 164.00 & 240.00 & 300.00 & 981.00 & 999.00 \\
\hline $\mathrm{APV}(\mathrm{hPa}) ; 8 \mathrm{pm}$ & 11.62 & 8.33 & -24.00 & -7.16 & 3.00 & 7.00 & 11.00 & 16.00 & 21.00 & 37.00 & 103.00 \\
\hline Precipitation (mm); $8 \mathrm{pm}$ & 0.88 & 4.53 & 0.00 & 0.00 & 0.00 & 0.00 & 0.00 & 0.01 & 1.00 & 19.16 & 101.00 \\
\hline DPT $\left({ }^{\circ} \mathrm{C}\right) ; 8 \mathrm{pm}$ & 10.98 & 10.26 & -23.50 & -13.02 & -3.20 & 3.30 & 12.00 & 19.90 & 24.00 & 26.50 & 28.90 \\
\hline Visibility $(\mathrm{km}) ; 8 \mathrm{pm}$ & 9.03 & 4.59 & 0.00 & 1.50 & 3.00 & 5.00 & 10.00 & 12.00 & 15.00 & 20.00 & 25.00 \\
\hline Temperature $\left({ }^{\circ} \mathrm{C}\right) ; 8 \mathrm{pm}$ & 16.99 & 9.37 & -5.20 & -1.42 & 3.50 & 9.00 & 18.30 & 25.00 & 28.70 & 32.62 & 35.80 \\
\hline STEMI; total & 0.12 & 0.36 & 0.00 & 0.00 & 0.00 & 0.00 & 0.00 & 0.00 & 1.00 & 1.00 & 3.00 \\
\hline SLP (hPa); total & 177.74 & 135.24 & 0.00 & 7.66 & 45.00 & 86.00 & 165.00 & 242.00 & 303.00 & 981.00 & 999.00 \\
\hline APV $(\mathrm{hPa})$; total & -0.24 & 15.20 & -62.00 & -36.00 & -21.00 & -11.00 & 2.00 & 11.00 & 17.00 & 32.00 & 103.00 \\
\hline Precipitation (mm); total & 0.83 & 4.34 & 0.00 & 0.00 & 0.00 & 0.00 & 0.00 & 0.00 & 0.90 & 19.00 & 101.00 \\
\hline DPT $\left({ }^{\circ} \mathrm{C}\right)$; total & 10.73 & 10.39 & -23.50 & -13.50 & -3.60 & 2.80 & 11.80 & 19.80 & 23.90 & 26.20 & 29.50 \\
\hline Visibility $(\mathrm{km})$; total & 8.13 & 4.72 & 0.00 & 0.90 & 2.50 & 4.00 & 7.00 & 12.00 & 15.00 & 20.00 & 30.00 \\
\hline Temperature $\left({ }^{\circ} \mathrm{C}\right)$; total & 16.76 & 9.83 & -7.40 & -3.40 & 2.80 & 8.50 & 18.10 & 24.80 & 28.80 & 34.60 & 40.00 \\
\hline
\end{tabular}

\section{Statistical analyses}

This was a time series study. Generalized additive Poisson models were utilized to plot numbers of patients with STEMI within 6 hour intervals against climatological variations after accounting for the effects of the hour and season [8]. All analyses were performed using R version 3.0.2 (http://www.Rproject.org). The R package, mgcv, was used to fit the GAMs in conjunction with the dlnm package, which was used for distributed lag modeling. Long-term time trends were controlled using a smooth function for each 6 hour interval of study, and seasonality was controlled using a smooth function for the day of the year, with a maximum of $4 \mathrm{df}$. Meteorological parameters were initially modeled using non-linear polynomial-constrained distributed lag models, with a maximum lag of 30 . The $\mathrm{df}$ for the lag structure was 4 , and the $\mathrm{df}$ for each smooth term describing the association between the predictor and the response was 4. The 'quasipoisson' option was used to account for the possibility of overdispersion (variance greater than the mean) of outcome variables. These choices were based on the results of previous studies [5]. The meteorological variables were included in the model simultaneously; however, two of the variables, dew point temperature and temperature, appeared to be strongly correlated and were not included in the model together; therefore, the variable of temperature was included in the model to allow comparisons with other studies.

After the core model was built, we plotted the relationship between meteorological conditions and the onset of STEMI. Because a unit change in meteorological conditions above or below a particular threshold would have led to different effect estimates with the DLNMs, we calculated each meteorological parameter's risk using a cutoff (percentile) value and compared this value to another cutoff value over lags $0-30$. Therefore, the relative risks $(R R S)$ and 95\% confidence intervals $(C I)$ for the onset of STEMI at the first percentile, 10 th percentile, 25 th percentile, 75 th percentile, 90 th percentile, and 99 th percentile of meteorological conditions were calculated and compared with the 50th percentile of meteorological conditions. The selection of these cutoffs for calculating the RRs and 95\% CIs was made based on a previous study [9]. 
Jia et al.: Meteorological Parameters and STEMI

Table 2. Relative risks and corresponding 95\% confidence intervals of sea level pressure (hPa) for each 6 hour interval of STEMI over multiple lags, during the study period (from 1 January 2003 to 31 December 2010)

\begin{tabular}{|c|c|c|c|c|c|c|}
\hline Lag & $\begin{array}{l}\text { 1stpercentile(7.60) vs } \\
\text { 50thpercentile }(165.00)\end{array}$ & $\begin{array}{l}\text { 10thpercentile }(45.00) \text { vs } \\
50 \text { thpercentitle }(165.00)\end{array}$ & $\begin{array}{l}\text { 25thpercentile( }(86000) \mathrm{vs} \\
\text { 50thpercentle }(165.00)\end{array}$ & $\begin{array}{l}\text { 75thpercentile }(24200) \mathrm{vs} \\
50 \text { thpercentile }(165.00)\end{array}$ & $\begin{array}{l}\text { 90thpercentile }(303.00) \mathrm{vs} . \\
50 \text { thperventile }(165.00)\end{array}$ & $\begin{array}{l}\text { 99thpercentile }(98100) \text { vs } \\
\text { 50thperentile }(165.00)\end{array}$ \\
\hline 0 & $105(0.76 \sim 145)$ & $104(0.81 \sim 134)$ & $103(0.85 \sim 1.26)$ & $0.91(0.79 \sim 105)$ & $0.85(0.66 \sim 109)$ & $0.98(0.63 \sim 1.52)$ \\
\hline $0-1$ & $107(0.76 \sim 149)$ & $101(0.78 \sim 131)$ & $0.98(0.80 \sim 1.21)$ & $101(0.87 \sim 1.15)$ & $101(0.78 \sim 131)$ & $0.89(0.54 \sim 143)$ \\
\hline $0-3$ & $1.10(0.77 \sim 1.58)$ & $104(0.79 \sim 138)$ & $101(0.81 \sim 126)$ & $0.95(0.81 \sim 1.11)$ & $0.90(0.68 \sim 1.20)$ & $0.78(0.44 \sim 1.39)$ \\
\hline $0-7$ & $113(0.75 \sim 170)$ & $107(0.78 \sim 140)$ & $102(0.79 \sim 131)$ & $102(0.86 \sim 121)$ & $103(0.76 \sim 141)$ & $0.83(0.42 \sim 165)$ \\
\hline $0-14$ & $0.89(0.55 \sim 144)$ & $0.93(0.65 \sim 134$ & $0.97(0.73 \sim 129)$ & $100(0.83 \sim 123)$ & $101(0.72 \sim 144)$ & $1.24(0.54 \sim 283)$ \\
\hline $0-21$ & $0.73(0.43 \sim 1.25)$ & $0.79(0.53 \sim 1.18)$ & $0.87(0.643 \sim 1.19)$ & $107(0.86 \sim 132)$ & $110(0.75 \sim 161)$ & $0.94(0.36 \sim 247)$ \\
\hline $0-30$ & $0.60(0.32 \sim 111)$ & $0.63(0.41 \sim 0.98)$ & $0.75(0.50 \sim 103)$ & $107(0.85 \sim 134)$ & $108(0.72 \sim 162)$ & $105(0.35 \sim 3.17)$ \\
\hline
\end{tabular}

Table 3. Relative risks and corresponding $95 \%$ confidence intervals of atmospheric pressure variations during the previous three hours (hPa) for each 6 hour interval of STEMI over multiple lags, during the study period (from 1 January 2003 to 31 December 2010)

\begin{tabular}{|c|c|c|c|c|c|c|}
\hline Lag & $\begin{array}{l}\text { 1stpercentile }(-36.00) \mathrm{vs} \\
\text { 50thpercentile(200) }\end{array}$ & $\begin{array}{l}\text { 10thpercentile }(-2100) \mathrm{vs} \\
50 \text { thpercentile }(200)\end{array}$ & $\begin{array}{l}\text { 25thpercentile }(-1100) \mathrm{vs} \\
50 \text { thpercentile }(200)\end{array}$ & $\begin{array}{l}\text { 75thpercentile(1100)vs } \\
\text { 50thpercentitle(200) }\end{array}$ & $\begin{array}{l}90 \text { thpercentile }(17.00) \mathrm{vs} \\
50 \text { thperwentile( }(200)\end{array}$ & $\begin{array}{l}\text { 99thpercentile(3200) vs } \\
50 \text { thpercentile(200) }\end{array}$ \\
\hline 0 & $166(136 \sim 203)$ & $147(130 \sim 167)$ & $122(1.12 \sim 133)$ & $116(107 \sim 125)$ & $127(1.13 \sim 143)$ & $116(0.92 \sim 146)$ \\
\hline $0-1$ & $127(0.87 \sim 185)$ & $145(1.13 \sim 185)$ & $128(108 \sim 152)$ & $103(0.91 \sim 1.17)$ & $104(0.85 \sim 127)$ & $083(0.59 \sim 117)$ \\
\hline $0-3$ & $0.40(0.19 \sim 0.85)$ & $102(0.63 \sim 163)$ & $120(0.89 \sim 163)$ & $0.83(0.70 \sim 0.99)$ & $0.73(0.55 \sim 0.97)$ & $0.55(0.34 \sim 0.89)$ \\
\hline 0.7 & $0.56(0.17 \sim 190)$ & $163(0.77 \sim 3.47)$ & $163(101 \sim 264)$ & $0.92(0.66 \sim 1.23)$ & $0.90(0.56 \sim 145)$ & $081(0.35 \sim 187)$ \\
\hline $0-14$ & $0.28(0.05 \sim 160)$ & $0.84(0.29 \sim 244)$ & $108(0.55 \sim 2.13)$ & $0.90(0.60 \sim 137)$ & $0.86(0.44 \sim 170)$ & $0.91(0.27 \sim 3.07)$ \\
\hline $0-21$ & $0.96(0.11 \sim 8.62)$ & $0.56(0.14 \sim 220)$ & $0.65(0.28 \sim 1.53)$ & $088(0.50 \sim 140)$ & $0.72(0.31 \sim 168)$ & $0.74(0.16 \sim 3.36)$ \\
\hline $0-30$ & $0.70(0.05 \sim 9.77)$ & $0.50(0.09 \sim 270)$ & $0.65(0.23 \sim 186)$ & $0.64(0.34 \sim 120)$ & $0.48(0.17 \sim 134)$ & $0.52(0.08 \sim 3.24)$ \\
\hline
\end{tabular}

Table 4. Relative risks and corresponding $95 \%$ confidence intervals of dew-point temperature $\left({ }^{\circ} \mathrm{C}\right)$ for each 6 hour interval of STEMI over multiple lags, during the study period (from 1 January 2003 to 31 December 2010)

\begin{tabular}{|c|c|c|c|c|c|c|}
\hline Lag & $\begin{array}{l}\text { 1stpercentile }(-1350) v s \\
50 \text { thpercentle }(1180)\end{array}$ & $\begin{array}{c}\text { 10thperøentile }(-3.60) \mathrm{vs} . \\
50 \text { thperøentile }(1180)\end{array}$ & $\begin{array}{c}\text { 25thpercentile }(280) \mathrm{vs} \\
50 \text { thpercentile }(1180)\end{array}$ & $\begin{array}{l}\text { 75thpercentile }(1980) v s . \\
\text { 50thpercentile }(1180)\end{array}$ & $\begin{array}{c}90 \text { thpercentile }(23.90) \mathrm{vs} \\
50 \text { thpercentile }(1180)\end{array}$ & $\begin{array}{l}\text { 99thperøentile }(2620) v s . \\
50 \text { thpercentile }(1180)\end{array}$ \\
\hline 0 & $0.72(0.23 \sim 2.22)$ & $0.96(0.73 \sim 1.26)$ & $0.99(0.80 \sim 1.19)$ & $0.54(0.15 \sim 193)$ & $0.52(0.12 \sim 227)$ & $0.52(0.11 \sim 251)$ \\
\hline $0-1$ & $0.70(0.23 \sim 216)$ & $0.96(0.73 \sim 1.26)$ & $0.97(0.80 \sim 1.18)$ & $0.53(0.15 \sim 187)$ & $0.50(0.12 \sim 220)$ & $0.50(0.10 \sim 243)$ \\
\hline $0-3$ & $0.65(021 \sim 200)$ & $0.94(0.72 \sim 1.24)$ & $0.98(0.80 \sim 1.20)$ & $0.54(0.15 \sim 194)$ & $0.53(0.12 \sim 230)$ & $0.53(0.11 \sim 256)$ \\
\hline $0-7$ & $0.62(023 \sim 222)$ & $0.96(0.71 \sim 1.23)$ & $0.98(0.80 \sim 1.20)$ & $0.55(0.15 \sim 197)$ & $0.55(0.12 \sim 239)$ & $0.56(0.11 \sim 269)$ \\
\hline $0-14$ & $064(0.20 \sim 192)$ & $0.95(0.72 \sim 1.25)$ & $0.97(0.80 \sim 1.19)$ & $0.55(0.15 \sim 195)$ & $0.54(0.12 \sim 237)$ & $0.55(0.11 \sim 267)$ \\
\hline $0-21$ & $0.74(0.24 \sim 235)$ & $0.98(0.74 \sim 1.21)$ & $0.995(0.78 \sim 182)$ & $0.51(0.14 \sim 182)$ & $0.49(0.11 \sim 2.18)$ & $0.50(0.10 \sim 243)$ \\
\hline $0-30$ & $0.61(0.19 \sim 193)$ & $0.93(0.78 \sim 1.23)$ & $100(0.82 \sim 123)$ & $0.65(0.18 \sim 234)$ & $0.65(0.15 \sim 287)$ & $0.66(0.13 \sim 3.24)$ \\
\hline
\end{tabular}

\section{Results}

Meteorological conditions and numbers of patients with STEMI within 6 hour intervals

Table 1 depicts the meteorological conditions and the numbers of subjects with STEMI during each 6 hour interval during the study period (from 1 January 2003 to 31 December 2010). A total of 1407 subjects with STEMI were included in our study population, and 0.12 subjects suffered a STEMI every 6 hours. During the study period, the median levels of meteorological conditions were 165.00, 2.00, 0.00, 11.80, 7.00 and 18.10 for sea level pressure ( $\mathrm{hPa}$, hectopascal), atmospheric pressure variations during the previous three hours ( $\mathrm{hPa}$, hectopascal), six hour precipitation (mm, millimeter), dew-point temperature $\left({ }^{\circ} \mathrm{C}\right.$, degrees Celsius), visibility ( $\mathrm{km}$, kilometer), and temperature $\left({ }^{\circ} \mathrm{C}\right.$, degrees Celsius), respectively. 
Jia et al.: Meteorological Parameters and STEMI

Table 5. Relative risks and corresponding 95\% confidence intervals of visibility $(\mathrm{km})$ for each 6 hour interval of STEMI over multiple lags, during the study period (from 1 January 2003 to 31 December 2010)

\begin{tabular}{|c|c|c|c|c|c|c|}
\hline Lag & $\begin{array}{l}\text { 1stperwentile }(0.90) \mathrm{vs} \\
50 \text { thpercentile }(7.00)\end{array}$ & $\begin{array}{l}\text { 10thperwentile }(250) \mathrm{vs} \\
50 \text { thpercentile }(7.00)\end{array}$ & $\begin{array}{l}\text { 25thperwentile }(4.00) \mathrm{vs} \\
50 \text { thpercentile }(7.00)\end{array}$ & $\begin{array}{c}\text { 75thperwentile }(12.00) \mathrm{vs} \\
50 \text { thperwentile }(7.00)\end{array}$ & $\begin{array}{l}90 \text { thperwentile }(15.00) \mathrm{vs} \text {. } \\
50 \text { thpercentile }(7.00)\end{array}$ & $\begin{array}{c}99 \text { th perwentile }(20.00) \mathrm{vs} \\
50 \text { thpercentile }(7.00)\end{array}$ \\
\hline 0 & $0.95(0.77 \sim 1.16)$ & $100(0.89 \sim 133)$ & $102(0.91 \sim 1.14)$ & $0.94(0.85 \sim 103)$ & $0.94(0.82 \sim 109)$ & $108(0.88 \sim 131)$ \\
\hline $0-1$ & $1.16(0.92 \sim 147)$ & $1.10(0.94 \sim 1.28)$ & $107(0.92 \sim 123)$ & $0.97(0.85 \sim 1.10)$ & $0.98(0.82 \sim 118)$ & $107(083 \sim 138)$ \\
\hline $0-3$ & $106(0.78 \sim 143)$ & $0.90(0.74 \sim 111)$ & $0.89(0.74 \sim 109)$ & $0.94(0.80 \sim 111)$ & $0.98(0.78 \sim 1.23)$ & $138(101 \sim 188)$ \\
\hline $0-7$ & $0.91(0.62 \sim 134)$ & $085(0.65 \sim 1.11)$ & $0.86(0.67 \sim 112)$ & $0.80(0.64 \sim 0.99)$ & $082(0.61 \sim 110)$ & $149(101 \sim 219)$ \\
\hline $0-14$ & $1.18(0.72 \sim 191)$ & $0.86(0.61 \sim 123)$ & $084(0.59 \sim 118)$ & $0.85(0.64 \sim 113)$ & $0.89(0.61 \sim 132)$ & $160(0.97 \sim 260)$ \\
\hline $0-21$ & $106(0.59 \sim 190)$ & $0.87(0.54 \sim 133)$ & $0.87(0.57 \sim 131)$ & $0.79(0.56 \sim 110)$ & $0.82(0.51 \sim 130)$ & $159(086 \sim 292)$ \\
\hline $0-30$ & $0.97(0.48 \sim 197)$ & $089(0.54 \sim 146)$ & $0.90(0.55 \sim 146)$ & $0.78(0.52 \sim 1.17)$ & $080(0.46 \sim 137)$ & $142(0.69 \sim 292)$ \\
\hline
\end{tabular}

Table 6. Relative risks and corresponding 95\% confidence intervals of temperature $\left({ }^{\circ} \mathrm{C}\right)$ for each 6 hour period of STEMI over multiple lags, during the study period (from 1 January 2003 to 31 December 2010)

\begin{tabular}{|c|c|c|c|c|c|c|}
\hline Lag & $\begin{array}{l}\text { 1stpercentile }(-3.40) \mathrm{vs} \\
\text { 50thpercentilie(18.10) }\end{array}$ & $\begin{array}{l}\text { 10thpercentile }(280) \mathrm{vs} . \\
50 \text { thpercentile }(18.10)\end{array}$ & $\begin{array}{l}\text { 25thperwentile }(850) \text { vs } \\
\text { 50thperwentile }(1810)\end{array}$ & $\begin{array}{c}\text { T5thpercentle(24.80)vs. } \\
50 \text { thpercentile(18.10) }\end{array}$ & $\begin{array}{l}\text { 90thpercentile(2880)vs } \\
\text { 50thpercentitle(1810) }\end{array}$ & $\begin{array}{c}99 \text { thperwentile }(34.60) \text { vs } \\
\text { 50thpercentile(18.10) }\end{array}$ \\
\hline 0 & $058(0.40 \sim 083)$ & $0.60(0.46 \sim 0.78)$ & $0.69(0.57 \sim 0.83)$ & 133(1.14 156) & $139(1.13 \sim 171)$ & $117(0.84 \sim 163)$ \\
\hline $0-1$ & $129(0.84 \sim 197)$ & $104(0.75 \sim 145)$ & $0.94(0.75 \sim 1.18)$ & $125(102 \sim 153)$ & $129(0.99 \sim 169)$ & $109(0.72 \sim 165)$ \\
\hline $0-3$ & $119(0.71 \sim 199)$ & $0.94(0.63 \sim 140)$ & $0.89(0.67 \sim 1.18)$ & $100(0.76 \sim 131)$ & $105(0.74 \sim 148)$ & $130(0.76 \sim 221)$ \\
\hline $0-7$ & $125(0.71 \sim 220)$ & $101(0.66 \sim 157)$ & $0.95(0.70 \sim 129)$ & $100(0.74 \sim 136)$ & $114(0.77 \sim 169)$ & $182(101 \sim 327)$ \\
\hline $0-14$ & $207(1.111 \sim 386)$ & $141(0.87 \sim 228)$ & $114(0.81 \sim 160)$ & $0.86(0.61 \sim 123)$ & $0.98(0.63 \sim 1.53)$ & $181(0.93 \sim 3.53)$ \\
\hline $0-21$ & $165(0.84 \sim 3.24)$ & $137(0.82 \sim 229)$ & $118(0.81 \sim 170)$ & $0.97(0.66 \sim 143)$ & $123(0.75 \sim 201)$ & $290(137 \sim 6.13)$ \\
\hline $0-30$ & $160(0.79 \sim 3.25)$ & $1.12(0.65 \sim 193)$ & $0.98(0.67 \sim 146)$ & $087(0.56 \sim 133)$ & $107(0.62 \sim 184)$ & $269(117 \sim 6.17)$ \\
\hline
\end{tabular}

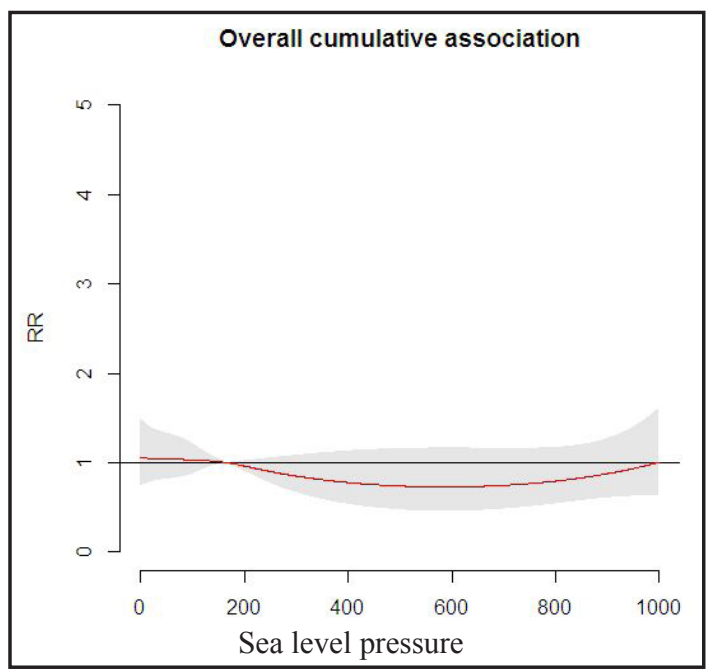

Fig. 1. The cumulative effects over lag 0 of sea level pressure (hPa, hectopascal) on the occurrence of STEMI.

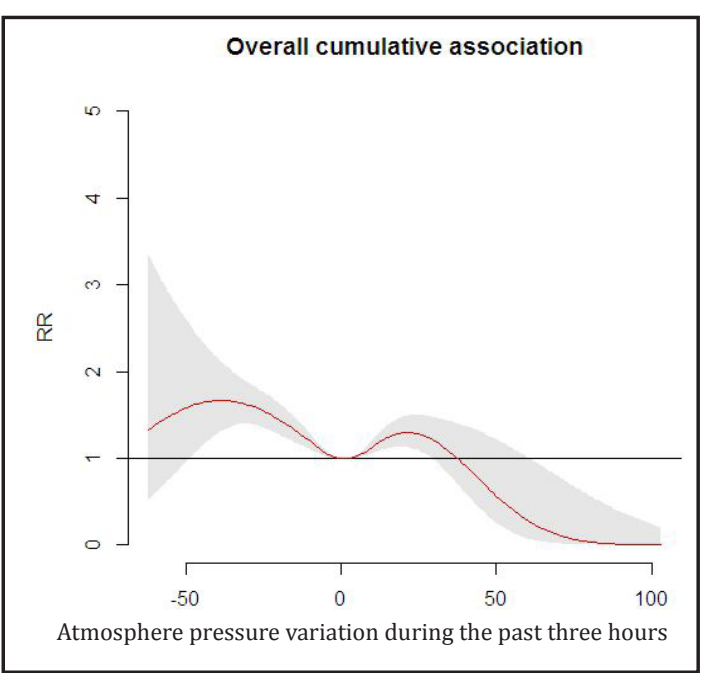

Fig. 2. The cumulative effects over lag 0 of atmospheric pressure variations during the previous three hours (hPa, hectopascal) on the occurrence of STEMI.

Generalized additive Poisson models for the time series

Generalized additive Poisson models were utilized to plot the results of the time series following adjustments for long-term trends: the results are included in Table 2-6, and the cumulative effects of meteorological conditions on the onset of STEMI for each 6 hour period at lag 0 are presented in Figure 1-6. A relationship between meteorological conditions, including sea level pressure ( $\mathrm{hPa}$, hectopascal), six hour precipitation ( $\mathrm{mm}$, millimeter), dew-point temperature $\left({ }^{\circ} \mathrm{C}\right.$, degrees Celsius), and visibility ( $\mathrm{mm}$, kilometer), and the onset of STEMI was not observed in this study; however, atmospheric pressure (hPa, hectopascal) variations during the previous three hours and temperature $\left({ }^{\circ} \mathrm{C}\right.$, degrees Celsius) were each significantly associated with the occurrence of STEMI within each 6 hour period. 


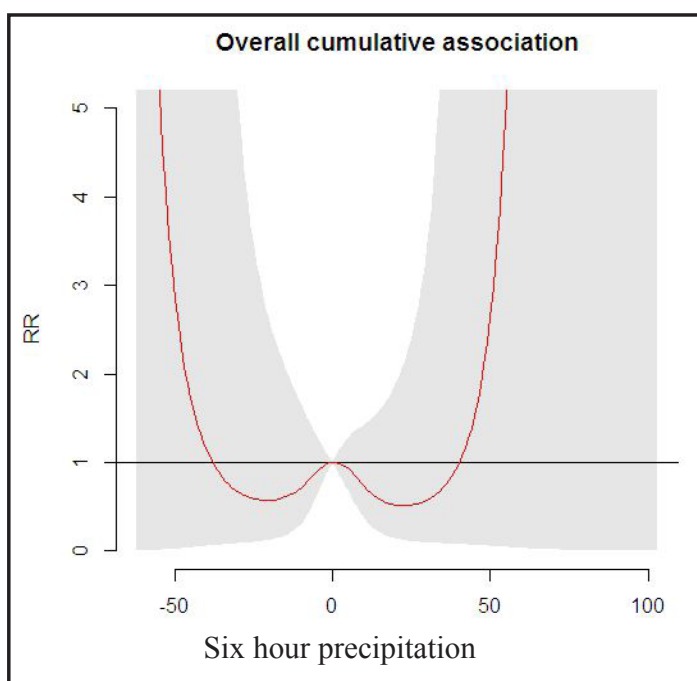

Fig. 3. The cumulative effects over lag 0 of six hour precipitation ( $\mathrm{mm}$, millimeter) on the occurrence of STEMI.

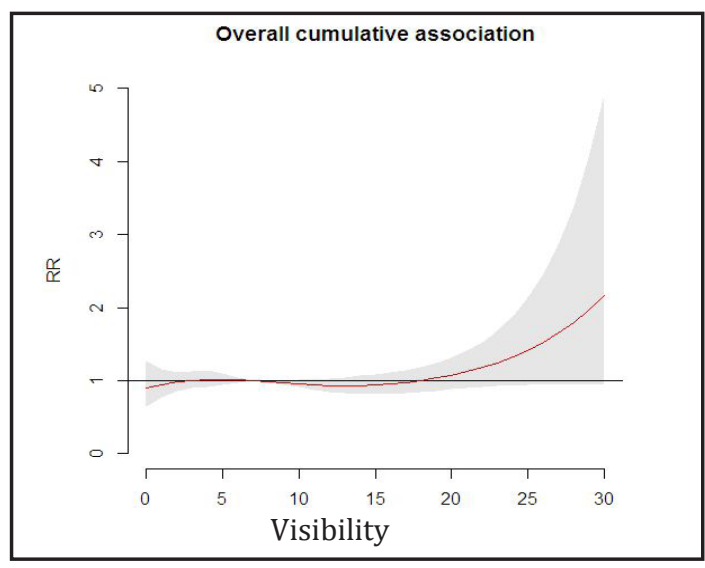

Fig. 5. The cumulative effects over lag 0 of visibility (km, kilometer) on the occurrence of STEMI.

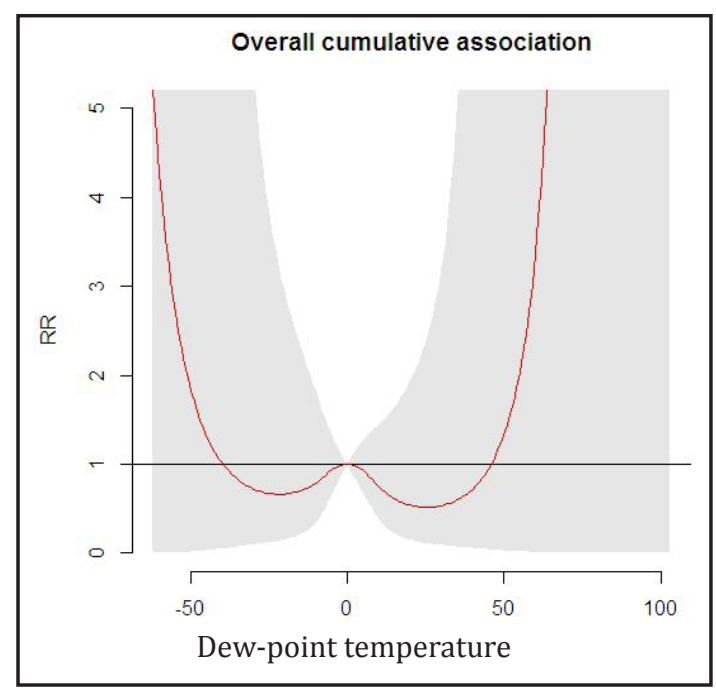

Fig. 4. The cumulative effects over lag 0 of dew-point temperature $\left({ }^{\circ} \mathrm{C}\right.$, degrees Celsius) on the occurrence of STEMI.

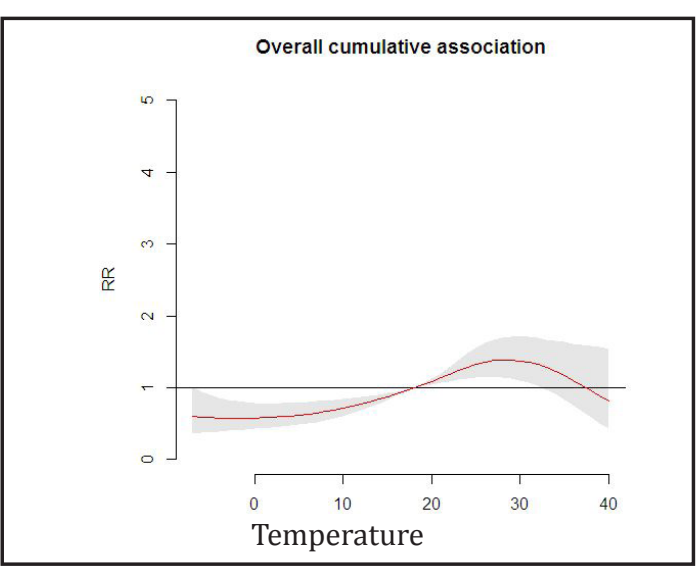

Fig. 6. The cumulative effects over lag 0 of temperature $\left({ }^{\circ} \mathrm{C}\right.$, degrees Celsius) on the occurrence of STEMI.

The relationship between atmospheric pressure variations during the previous three hours and STEMI

Figure 2 shows the cumulative effects of atmospheric pressure variations during the previous three hours (hPa, hectopascal) on the occurrence of STEMI for each 6 hour interval at lag 0 . The atmospheric pressure variation-morbidity of STEMI relationship curve was U-shaped and illustrated a higher risk of STEMI at both high and low atmospheric pressures variations . As indicated in Table 3, the effects of a decrease in atmospheric pressure lasted longer than 6 hours (Lag 0-1); however, the effects of an increase in atmosphere pressure variations were limited to the first 6 hours (Lag 0). At lag 0, the $R R s(95 \% C I)$ for the first percentile, 10th percentile, 25th percentile, 75th percentile, 90th percentile, and 99th percentile for atmospheric pressure variation were 1.66 (1.36 2.03), 1.47 (1.30 1.67), 1.22 (1.12 1.33), 1.16 (1.07 1.25), 1.27 (1.13 1.43), and 1.16 (0.92 1.46), respectively, compared with the 50 th percentile for atmospheric pressure variation

The relationship between temperature and STEMI

Figure 6 and Table 6 each show the relationship between temperature within each 6 hour interval and the occurrence of STEMI. The cumulative effects of temperature on the 
occurrence of STEMI suggest that increases in temperature are a risk factor for STEMI and that the effects of such an event last longer than 6 hours (Lag 0-1). At lag 0, the RRs $(95 \%$ $C I$ ) for the first percentile, 10th percentile, and 25th percentile were $0.58(0.40 \sim 0.83)$, 0.60 (0.46 0.78), 0.69 (0.57 0.83), respectively, compared to the 50th percentile for temperature. Compared to the 50th percentile for temperature, the $R R s(95 \% C I)$ for the 75th percentile, 90th percentile, and 99th percentile for temperature were 1.33 (1.14 1.56), 1.39 (1.13 1.71), and $1.17(0.84 \sim 1.63)$, respectively. At lag 0-7, the $R R(95 \% C I)$ for the 99th percentile for temperature was 1.82 (1.01 3.27), compared to the 50th percentile for temperature. At lag $0-14$, the $R R(95 \% C l)$ for the 1st percentile for temperature was 2.07 (1.11 3.86), compared to the 50th percentile for temperature.

\section{Discussion}

To the best of our knowledge, this is the first study to explore the association between meteorological parameters and STEMI occurrence in China. Based on the eight-year, singlecenter study, significant relationships among the onset of STEMI and atmospheric pressure variations during the past three hours and temperature were observed after accounting for long-term time trends.

Recently, numerous studies have examined short-term associations between ambient temperature and acute myocardial infarction, with inconsistent results. A case-crossover study from England and the Wales Myocardial Ischaemia National Audit Project (MINAP) database suggests that with each $1^{\circ} \mathrm{C}$ increase in temperature above $20^{\circ} \mathrm{C}$, the risk of ST elevation myocardial infarction is increased by $2.7 \%$ (0.6\% to $4.9 \%$ ) within one to six hours following exposure [10]. The data were based on hourly observations. After accounting for the day of the week, long-term trends and seasonal effects, a $1^{\circ} \mathrm{C}$ drop below a threshold temperature of $24^{\circ} \mathrm{C}$ was significantly $(\mathrm{p}<0.0001)$ associated with an AMI hospitalizationrisk increase of 3.7\% (average lag 0-13 temperature) in Hong Kong, 2.6\% (average lag $0-15$ ) in Taipei, and $4.0 \%$ (average lag 0-11) in Kaohsiung. No significant heat effects were observed. However, the above results were based on daily data regarding AMI hospitalizations and mean temperatures, which were collected between 2000 and 2009 in three warmclimate Asian cities [9]. In the present study, both temperature data and data regarding the occurrence of STEMI were collected at 6 hour intervals from 2003 to 2010, and the results indicated that although the immediate association between temperature and STEMI (lag 0 ) is consistently positive (higher temperatures are associated with a higher incidence of STEMI) long-term associations (lags 0-7, lags 0-14) appear as a U-shaped curve, as both high and low temperatures are associated with a higher risk of STEMI. The results of the present study are consistent with those of MINAP, but they were not consistent with the results of studies performed in three warm-climate Asian cities. The exact mechanism underlying the U-shaped association between temperature and STEMI requires further study.

During the past decade, various studies have noted the relationship between meteorological factors and cardiovascular mortality and morbidity. However, there are no studies exploring the relationship between atmospheric pressure variations and the occurrence of STEMI worldwide. To the best of our knowledge, this is the first study to suggest that atmospheric pressure variations, rather than the atmospheric pressure levels, increase the risk of STEMI. The exact mechanism underlying this association requires further study. In a study of diving fatalities, 947 recreational open-circuit scuba diving deaths that occurred between 1992 and 2003 were investigated, and the results indicate that cardiac incidents account for $26 \%$ of disabling injuries and $13 \%$ of deaths. The OR of disabling injury due to cardiovascular disease was 10.5 [11], and the results suggest that atmospheric pressure variations may be associated with cardiovascular disease.

Global climate change (GCC) is associated with significant changes in long-term weather characteristics and short-term weather extremes in different geographic regions [12]. The results of the present study may have important implications for the development of 
strategies and policies to prevent climate change-related STEMI. However, this was a singlecenter study conducted in China, and the conclusions drawn based on this study's results may not be applicable to other locations. Therefore, large-scale, multi-center epidemiological studies are necessary to determine the relationship between climate changes and STEMI. Furthermore, biological mechanisms concerning the relationship among the risk of STEMI, increases in temperature, and atmospheric pressure variations must be explored via experimental studies.

\section{Acknowledgements}

This study was supported by the National Natural Science Foundations of China (grants 30400173 and 30971257 ). A project funded by the Priority Academic Program Development of Jiangsu Higher Education Institutions

Dr. En-Zhi Jia is an Assistant Fellow at the Collaborative Innovation Center for Cardiovascular Disease Translational Medicine.

\section{References}

- O O'Gara PT, Kushner FG, Ascheim DD, Casey DE Jr, Chung MK, de Lemos JA, Ettinger SM, Fang JC, Fesmire FM, Franklin BA, Granger CB, Krumholz HM, Linderbaum JA, Morrow DA, Newby LK, Ornato JP, Ou N, Radford MJ, Tamis-Holland JE, Tommaso CL, Tracy CM, Woo YJ, Zhao DX, Anderson JL, Jacobs AK, Halperin JL, Albert NM, Brindis RG, Creager MA, DeMets D, Guyton RA, Hochman JS, Kovacs RJ, Kushner FG, Ohman EM, Stevenson WG, Yancy CW; American College of Cardiology Foundation/American Heart Association Task Force on Practice Guidelines: 2013 ACCF/AHA guideline for the management of ST-elevation myocardial infarction: a report of the American College of Cardiology Foundation/American Heart Association Task Force on Practice Guidelines. Circulation 2013;127:e362-425.

-2 Turkmen S, Dogdu O, Tekin K, Kucukdurmaz Z, Cagliyan CE, Sarikaya S, Yucel H, Karapinar H, Ozkan B, Uysal OK, Basara A, Sancaktar E, Yilmaz A: The relationship between neutrophil/lymphocyte ratio and the TIMI flow grade in patients with STEMI undergoing primary PCI. Eur Rev Med Pharmacol Sci 2013;17:21852189.

3 Madrigano J, Mittleman MA, Baccarelli A, Goldberg R, Melly S, von Klot S, Schwartz J: Temperature, myocardial infarction, and mortality: effect modification by individual- and area-level characteristics. Epidemiology 2013;24:439-446.

-4 Lee JH, Chae SC, Yang DH, Park HS, Cho Y, Jun JE, Park WH, Kam S, Lee WK, Kim YJ, Kim KS, Hur SH, Jeong MH; Korea Acute Myocardial Infarction Registry Investigators: Influence of weather on daily hospital admissions for acute myocardial infarction (from the Korea Acute Myocardial Infarction Registry). Int J Cardiol 2010;144:16-21.

-5 Goggins WB, Chan EY, Yang CY: Weather, pollution, and acute myocardial infarction in Hong Kong and Taiwan. Int J Cardiol 2013;168:243-249.

6 Jia EZ, Xu ZX, Cai HZ, Guo CY, Li L, Zhu TB, Wang LS, Cao KJ, Ma WZ, Yang ZJ: Time distribution of the onset of chest pain in subjects with acute ST-elevation myocardial infarction: an eight-year, single-center study in China. PLoS One 2012; 7:e32478.

-7 Thygesen K, Alpert JS, White HD; Joint ESC/ACCF/AHA/WHF Task Force for the Redefinition of Myocardial Infarction: Universal definition of myocardial infarction. Circulation 2007;116:2634-2653.

8 Wood SN: Generalized additive models: An introduction with R. Chapman and Hall/CRC, Oxford. 2006, pp 243-248.

-9 Chen R, Wang C, Meng X, Chen H, Thach TQ, Wong CM, Kan H: Both low and high temperature may increase the risk of stroke mortality. Neurology 2013;81:1064-1070.

10 Bhaskaran K, Armstrong B, Hajat S, Haines A, Wilkinson P, Smeeth L: Heat and risk of myocardial infarction: hourly level case-crossover analysis of MINAP database. BMJ 2012;345:e8050.

11 Denoble PJ, Caruso JL, Dear Gde L, Pieper CF, Vann RD: Common causes of open-circuit recreational diving fatalities. Undersea Hyperb Med 2008;35:393-406.

12 Balbus JM, Boxall AB, Fenske RA, McKone TE, Zeise L: Implications of global climate change for the assessment and management of human health risks of chemicals in the natural environment. Environ Toxicol Chem 2013;32:62-78. 